\section{(2) OPEN ACCESS}

\title{
Undetectable equals untransmittable $(U=U)$ : awareness and associations with health outcomes among people living with HIV in 25 countries
}

\author{
Chinyere Okoli, ${ }^{1}$ Nicolas Van de Velde, ${ }_{1}^{1}$ Bruce Richman, ${ }^{2}$ Brent Allan, ${ }^{3}$ \\ Erika Castellanos, ${ }^{4}$ Benjamin Young, ${ }^{5}$ Garry Brough, ${ }^{6}$ Anton Eremin 이, \\ Giulio Maria Corbelli, ${ }^{8}$ Marta Mc Britton, ${ }^{9}$ W. David Hardy, ${ }^{10}$ Patricia de los Rios ${ }^{5}$
}

\begin{abstract}
- Additional material is published online only. To view please visit the journal online (http://dx.doi.org/10.1136/ sextrans-2020-054551).
\end{abstract}

For numbered affiliations see end of article.

\section{Correspondence to} Dr Chinyere Okoli, ViiV Healthcare, Brentford TW8 9GS, UK; Chinyere.x.okoli@ viivhealthcare.com

Received 17 April 2020 Revised 4 June 2020 Accepted 14 June 2020 Published Online First 30 July 2020

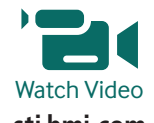

sti.bmj.com

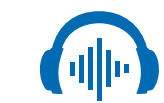

Listen to Podcast sti.bmj.com

Check for updates

\section{(c) Author(s) (or their} employer(s)) 2021. Re-use permitted under CC BY-NC. No commercial re-use. See rights and permissions. Published by BMJ.

\footnotetext{
To cite: Okoli C, Van de Velde N, Richman B, et al. Sex Transm Infect 2021:97:18-26.
}

\section{ABSTRACT}

Objectives 'Undetectable equals Untransmittable' $(U=U)$ is an empowering message that may enable people living with HIV (PLHIV) to reach and maintain undetectability. We estimated the percentage of PLHIV who ever discussed $\mathrm{U}=\mathrm{U}$ with their main HIV care provider, and measured associations with health-related outcomes. Secondarily, we evaluated whether the impact of the $U=U$ message varied between those who heard it from their healthcare provider (HCP) vs from elsewhere.

Methods Data were from the 25-country 2019 Positive Perspectives Survey of PLHIV on treatment ( $n=2389)$. PLHIV were classified as having discussed $U=U$ with their HCP if they indicated that their HCP had ever told them about $U=U$. Those who had not discussed $U=U$ with their HCP but were nonetheless aware that 'My HIV medication prevents me from passing on HIV to others' were classified as being made aware of $U=U$ from non-HCP sources. Multivariable logistic regression was used to measure associations between exposure to $\mathrm{U}=\mathrm{U}$ messages and health outcomes.

Results Overall, 66.5\% reported ever discussing $\mathrm{U}=\mathrm{U}$ with their HCP, from $38.0 \%$ (South Korea) to $87.3 \%$ (Switzerland). Prevalence was lowest among heterosexual men (57.6\%) and PLHIV in Asia (51.3\%). Compared with those unaware of $U=U$, those reporting $\mathrm{U}=\mathrm{U}$ discussions with their HCP had lower odds of suboptimal adherence $(A O R=0.59,95 \% \mathrm{Cl} 0.44$ to $0.78)$ and higher odds of self-reported viral suppression ( $A O R=2.34,95 \% \mathrm{Cl} 1.72$ to 3.20), optimal sexual health ( $A O R=1.48,95 \% \mathrm{Cl} 1.14$ to 1.92$)$ and reporting they 'always shared' their HIV status (AOR=2.99, 95\% $\mathrm{Cl} 1.42$ to 6.28). While exposure to $\mathrm{U}=\mathrm{U}$ information from non-HCP sources was beneficial too, the observed associations were attenuated relative to those seen with reported discussions with HCPs.

Conclusion HCP discussion of $U=U$ with PLHIV was associated with favourable health outcomes. However, missed opportunities exist since a third of PLHIV reported not having any $U=U$ discussion with their $\mathrm{HCP} . \mathrm{U}=\mathrm{U}$ discussions with PLHIV should be considered as a standard of care in clinical guidelines.

\section{INTRODUCTION}

Despite global targets to eliminate AIDS as a public health threat by 2030, about 14\% (3.5 million) of the 25.4 million people living with HIV (PLHIV) on treatment are virally non-suppressed. ${ }^{12}$ The evidence-based campaign, 'Undetectable Equals Untransmittable' $(\mathrm{U}=\mathrm{U}),^{3-8}$ is an empowering discussion that may help motivate and incentivise PLHIV to reach and maintain undetectability. ${ }^{2}$ The key message behind this informational campaign is that 'for people who achieve and maintain viral suppression, there is effectively no risk of transmitting HIV to their HIV-negative sexual partners'.

$\mathrm{U}=\mathrm{U}$ is appealing in its simplicity, person-focus and potential downstream benefits in promoting prevention at all levels, including primary (preventing transmission to uninfected persons), secondary (ensuring regular viral load monitoring and health screenings among PLHIV) and tertiary (improving PLHIV's quality of life). By focusing on being 'undetectable', an objectively measured health state, $\mathrm{U}=\mathrm{U}$ provides PLHIV with an unambiguous health target that emphasises personal responsibility. ${ }^{3} 4$ 'Untransmissibility', the reward for 'Undetectability' may be particularly appealing for serodiscordant couples in a relationship or wishing to start a family. Besides encouraging antiretroviral therapy (ART) initiation and adherence among PLHIV, U $=\mathrm{U}$ could also recalibrate social norms among non-HIV populations, given that transmission risk is a major source of fear/stigma. ${ }^{9-12}$

The $\mathrm{U}=\mathrm{U}$ message has been incorporated into numerous public health campaigns and clinical guidelines. $^{3413}$ Discussing $\mathrm{U}=\mathrm{U}$ in clinical settings is vital because patients perceive information they hear directly from their healthcare providers (HCPs) as being more believable. ${ }^{14}$ The impact of HCP counselling has been well demonstrated in other areas of public health such as smoking cessation. ${ }^{15}$ Since clinicians are likely the first professionals to whom a newly diagnosed person will turn for advice about their new health circumstance, it is essential to evaluate the extent to which HCPs share information on $\mathrm{U}=\mathrm{U}$ with their patients and whether this varies by recency of diagnosis, at-risk groups for HIV transmission and by burden of disease. ${ }^{16-21}$

This study therefore examined: (1) Prevalence and disparities in self-report of having had a $\mathrm{U}=\mathrm{U}$ discussion with HCPs. (2) Associations between exposure to $\mathrm{U}=\mathrm{U}$ information and health-related perceptions and behaviours. We further examined 
whether the observed impact of exposure to $\mathrm{U}=\mathrm{U}$ message was modified by the source of the information (HCP or non-HCP sources) as well as by PLHIV characteristics.

\section{METHODS}

\section{Data source}

Sample size

The 2019-2020 Positive Perspectives Study was fielded from 3 April 2019 to 31 January $2020(n=2389$, response rate $=58.6 \%)$. It was a web-based, self-reported, cross-sectional survey of PLHIV in 25 countries: USA $(n=400)$, South Africa $(n=179)$, Russia $(n=150)$, UK $(n=123)$, Australia $(n=120)$, Canada $(n=120)$, France $(n=120)$, Germany $(n=120)$, Italy $(n=120)$, Spain $(n=120)$, Japan $(n=75)$, Mexico $(n=63)$, Portugal $(n=60)$, Brazil $(n=58)$, Switzerland $(n=55)$, Taiwan $(n=55)$, Netherlands $(n=51)$, Argentina $(n=50)$, Austria $(n=50)$, Chile $(n=50)$, China $(n=50)$, Ireland $(n=50)$, Belgium $(n=50)$, Poland $(n=50)$, and South Korea $(n=50)$. Sampling was purposive and aimed at achieving adequate sample sizes within three prespecified strata: those diagnosed as HIV seropositive within the last 2 years, women and persons aged $50+$ years. The rationale was to better understand the specific perceptions and attitudes of these population subgroups who may sometimes be under-represented in routine surveillance.

\section{Survey recruitment/fielding}

Participants were eligible for selection if they were aged $\geq 18$ years, confirmed being HIV seropositive and were on ART. Of the 2389 participants, 1387 (58.1\%) were recruited from support groups/charities for patients with HIV; 627 (26.2\%) from existing panels of PLHIV; 204 (8.5\%) from local operating company contacts; 108 (4.5\%) from patient-to-patient referrals; and $63(2.6 \%)$ from social media (online supplementary table 1 ). The survey was fielded in 20 languages.

The questionnaire, developed specifically for this primary data collection, comprised 60 questions designed to be completed in 25-30 min. It was developed, piloted and reviewed alongside an advisory panel that included PLHIV. The survey was sponsored by ViiV Healthcare and fielded independently by Ipsos Healthcare. To minimise bias in administering the survey, there was double blinding: ViiV Healthcare was not identified to participants as the study sponsor; likewise, participants' identities were not revealed to ViiV Healthcare. Participants were however told the study was being conducted 'on behalf of a pharmaceutical company'.

\section{MEASURES}

\section{Exposure to $\mathrm{U}=\mathrm{U}$ information}

Conceptually, we classified participants into three categories based on exposure to $U=U$ information: (a) Not heard of $U=U$. (b) Discussed $U=U$ with their HCP. (c) Heard about $U=U$ only from sources other than an HCP. Operationally, those who affirmed in the survey ('agree' or 'strongly agree') that 'My provider has told me about "undetectable=untransmittable", were classified as (b) regardless of whether the discussion was patient-prompted or HCP initiated, and also regardless of whether PLHIV heard about $\mathrm{U}=\mathrm{U}$ from other sources as well; those not informed of $\mathrm{U}=\mathrm{U}$ by their HCP, but who nonetheless reported awareness that 'My HIV medication prevents me from passing on HIV to others' were classified as (c); all others who provided dissenting responses to the two survey questions above were classified as (a). The specific wording of the questions above are shown in online supplementary table 2 . Respondents were also asked their perceived comfort discussing with HCPs their 'concerns about the safety of others/preventing transmission'.

\section{Self-rated health}

Participants rated their sexual, physical, mental and overall health. Responses of 'Very good' or 'Good' (vs 'Neither good nor poor'/'Poor'/'Very poor') were classified as optimal.

\section{Sharing of HIV status}

Both components of social norms-descriptive (estimated prevalence) and injunctive (perceptions of acceptability)—can mediate the stigma experienced among PLHIV. ${ }^{22}$ For example, PLHIV might be unwilling to share their HIV status because they perceive very few other people within their social circle as living with HIV (descriptive norms), or because having HIV is judged by people within their social circle as being undesirable (injunctive norms). ${ }^{9} 23$

We assessed whether exposure to $\mathrm{U}=\mathrm{U}$ messages was associated with differential willingness to share HIV status with others. PLHIV were asked various questions to determine their perceived comfort 'with sharing (their) HIV status'; if they had 'ever hidden or disguised (their) HIV medication to avoid revealing (their) status', and perceived comfort discussing with HCPs their 'concerns about privacy and not disclosing (their) HIV status'. PLHIV further identified whom they had shared their HIV status with besides their primary HIV care providers.

\section{Other variables}

Suboptimal adherence was a report of at least one reason for missing ART for at least five times within the past month. Other assessed variables included age, gender, sexual orientation, location of PLHIV and of their primary HCP, commute time to HCP, relationship status, and duration of disease. Several other aspects of engagement with HCPs were assessed, including sharing of health information, involvement in treatment decisions/care and communication.

\section{Analyses}

Percentages were compared with $\chi^{2}$ tests. Associations between receipt of $U=U$ message and health outcomes were measured using logistic regression. The outcomes analysed were selfreported viral suppression, suboptimal adherence, self-rated health, and privacy-related perceptions and behaviours. Separate logistic regression analyses were conducted for each outcome, adjusting for gender, age, sexual orientation, geographical region, duration of disease, domicile (metropolitan or nonmetropolitan), education and ethnicity. Confounding variables were identified statistically (independently associated with exposure and outcomes) as well as with directed acyclic graphs. To assess for effect modification by PLHIV characteristics $(\geq 10 \%$ difference in effect measure), analyses were further stratified by age ( $<50$ or $\geq 50$ years) and gender (men or women). All statistical procedures were performed with R V.3.6.1.

\section{RESULTS}

Mean age was $41.2(\mathrm{SD}=12.2)$ years. Overall, $29.1 \%$ selfidentified as women, $70.7 \%$ were aged $<50$ years and $73.5 \%$ had $>$ high school. Among those diagnosed with HIV prior to $2017,60.1 \%(1106 / 1841)$ currently prioritised preventing transmission to a sexual partner, and 20.4\% (376/1841) currently prioritised having children. Black participants made up $13.6 \%$ $(275 / 2014)$ of all respondents with information on ethnicity, 
Table 1 Attitudes and perceptions towards preventing HIV transmission and the percentage that reported ever discussing U=U with their healthcare provider among people living with HIV in 25 countries, Positive Perspectives Study, 2019

\begin{tabular}{|c|c|c|c|c|c|c|}
\hline Characteristics & Categories & $\mathrm{N}, \%$ & $\begin{array}{l}\text { Comfortable } \\
\text { discussing HIV } \\
\text { transmission with } \\
\text { HCP, \% }\end{array}$ & $\begin{array}{l}\text { Ever discussed } \mathrm{U}=\mathrm{U} \\
\text { with } \mathrm{HCP}, \%\end{array}$ & $\begin{array}{l}\text { Aware that HIV } \\
\text { medications prevent } \\
\text { HIV transmission, \% }\end{array}$ & $\begin{array}{l}\text { Either discussed } \mathrm{U}=\mathrm{U} \text { with } \\
\mathrm{HCP} \text { or reported awareness } \\
\text { that HIV medications prevent } \\
\text { transmission, \% }\end{array}$ \\
\hline Total & Overall & $2389(100.0)$ & 59.8 & 66.5 & 74.2 & 87.6 \\
\hline \multirow[t]{2}{*}{ Age, years } & $<50$ & $1690(70.7)$ & 55.5 & 65.5 & 74.0 & 87.0 \\
\hline & $50+$ & $699(29.3)$ & 70.1 & 68.8 & 74.7 & 89.0 \\
\hline \multirow[t]{3}{*}{ Gender } & Men & $1623(67.9)$ & 61.5 & 66.6 & 76.8 & 88.4 \\
\hline & Women & $696(29.1)$ & 58.0 & 64.7 & 67.8 & 85.1 \\
\hline & Other & $70(2.9)$ & 37.1 & 81.4 & 78.6 & 95.7 \\
\hline \multirow[t]{5}{*}{ Gender/sexual orientation } & Men who have sex with men & $1018(42.6)$ & 70.3 & 70.5 & 83.2 & 92.7 \\
\hline & $\begin{array}{l}\text { Men who have sex with } \\
\text { women }\end{array}$ & $479(20.1)$ & 42.8 & 57.6 & 63.0 & 78.7 \\
\hline & $\begin{array}{l}\text { Women who have sex with } \\
\text { men }\end{array}$ & $481(20.1)$ & 59.3 & 64.2 & 69.9 & 85.7 \\
\hline & $\begin{array}{l}\text { Women who have sex with } \\
\text { women }\end{array}$ & $62(2.6)$ & 45.2 & 61.3 & 54.8 & 74.2 \\
\hline & Other/indeterminate & 349 (14.6) & 55.6 & 70.8 & 72.8 & 90.0 \\
\hline \multirow[t]{2}{*}{ Overall health } & Suboptimal & 1012 (42.4) & 50.5 & 61.4 & 69.1 & 83.8 \\
\hline & Optimal & $1377(57.6)$ & 66.6 & 70.2 & 78.0 & 90.4 \\
\hline \multirow[t]{2}{*}{ Physical health } & Suboptimal & 953 (39.9) & 50.2 & 59.8 & 67.1 & 83.0 \\
\hline & Optimal & $1436(60.1)$ & 66.2 & 70.9 & 79.0 & 90.7 \\
\hline \multirow[t]{2}{*}{ Mental health } & Suboptimal & $1013(42.4)$ & 50.9 & 58.8 & 68.3 & 81.9 \\
\hline & Optimal & $1376(57.6)$ & 66.3 & 72.1 & 78.6 & 91.8 \\
\hline \multirow[t]{2}{*}{ Sexual health } & Suboptimal & $1227(51.4)$ & 55.7 & 63.0 & 70.9 & 85.8 \\
\hline & Optimal & $1162(48.6)$ & 64.1 & 70.1 & 77.7 & 89.5 \\
\hline \multirow[t]{6}{*}{ Geographical Region* } & Northern America & $520(21.8)$ & 53.8 & 67.3 & 64.2 & 84.8 \\
\hline & Europe & $1119(46.8)$ & 61.1 & 68.4 & 78.8 & 90.5 \\
\hline & Latin America & $221(9.3)$ & 59.7 & 60.6 & 71.9 & 84.6 \\
\hline & Asia & $230(9.6)$ & 50.4 & 51.3 & 64.3 & 78.7 \\
\hline & Australia & $120(5.0)$ & 81.7 & 80.0 & 90.8 & 97.5 \\
\hline & South Africa & $179(7.5)$ & 65.9 & 69.8 & 78.8 & 86.0 \\
\hline \multirow[t]{3}{*}{ Housing status } & Own & $722(30.2)$ & 61.1 & 65.7 & 73.1 & 88.4 \\
\hline & Rent & $916(38.3)$ & 63.3 & 68.1 & 77.5 & 89.2 \\
\hline & Other & 751 (31.4) & 54.2 & 65.2 & 71.2 & 85.0 \\
\hline \multirow[t]{2}{*}{ HCP location } & Metropolitan & $1583(66.3)$ & 64.8 & 69.0 & 79.7 & 90.9 \\
\hline & Non-metropolitan & $806(33.7)$ & 49.9 & 61.5 & 63.4 & 81.1 \\
\hline \multirow[t]{2}{*}{ PLHIV location } & Metropolitan & $1335(55.9)$ & 63.4 & 67.9 & 80.0 & 90.7 \\
\hline & Non-metropolitan & $1054(44.1)$ & 55.1 & 64.6 & 66.9 & 83.7 \\
\hline \multirow[t]{3}{*}{ Commute to $\mathrm{HCP}$} & $<30$ min & $869(36.4)$ & 65.5 & 71.9 & 78.3 & 91.4 \\
\hline & $30-59 \min$ & $1027(43.0)$ & 58.1 & 65.5 & 71.0 & 85.0 \\
\hline & $60+$ min/unknown & $493(20.6)$ & 53.1 & 58.8 & 73.8 & 86.4 \\
\hline \multirow[t]{2}{*}{ Employment status } & Employed & $1653(69.2)$ & 60.0 & 65.8 & 73.7 & 87.6 \\
\hline & Non-employed & $736(30.8)$ & 59.4 & 68.1 & 75.3 & 87.6 \\
\hline \multirow[t]{3}{*}{ Education } & $\leq$ High school & $532(22.3)$ & 59.0 & 72.6 & 72.0 & 88.5 \\
\hline & $>$ High school & $1756(73.5)$ & 61.4 & 63.7 & 74.7 & 87.0 \\
\hline & Refused to answer & $101(4.2)$ & 35.6 & 83.2 & 78.2 & 93.1 \\
\hline \multirow[t]{4}{*}{ Ethnicity } & White & $1393(58.3)$ & 61.7 & 67.1 & 74.3 & 88.4 \\
\hline & Black & $275(11.5)$ & 60.0 & 69.8 & 70.9 & 84.0 \\
\hline & Other & $346(14.5)$ & 59.0 & 63.9 & 74.0 & 86.7 \\
\hline & Unknown/missing & $375(15.7)$ & 53.3 & 64.0 & 76.5 & 88.3 \\
\hline \multirow{2}{*}{$\begin{array}{l}\text { Preventing HIV transmission was } \\
\text { a treatment goal when starting } \\
\text { treatment }\end{array}$} & No & $1216(50.9)$ & 57.6 & 61.8 & 69.4 & 84.7 \\
\hline & Yes & $1173(49.1)$ & 62.1 & 71.3 & 79.2 & 90.6 \\
\hline \multirow[t]{2}{*}{ Self-reported viral load } & Not suppressed/unknown & $619(25.9)$ & 48.5 & 59.6 & 54.3 & 79.2 \\
\hline & Suppressed & $1770(74.1)$ & 63.7 & 68.9 & 81.2 & 90.6 \\
\hline \multirow[t]{3}{*}{ HIV diagnosis year } & 2017-2019 & $548(22.9)$ & 55.5 & 67.3 & 67.9 & 88.5 \\
\hline & 2010-2016 & $913(38.2)$ & 54.2 & 66.2 & 73.1 & 85.0 \\
\hline & Pre-2010 & $928(38.8)$ & 67.8 & 66.3 & 79.1 & 89.7 \\
\hline
\end{tabular}

* Geographical regions were: Northern America (U.S. and Canada); Europe (Austria, Belgium, France, Germany, Italy, the Netherlands, Poland, Portugal, Ireland, Russia, Spain, Switzerland, and the UK); Latin America (Argentina, Brazil, Chile, and Mexico), and Asia (China, Japan, South Korea, and Taiwan); South Africa and Australia were analyzed separately.

$\mathrm{HCP}$, healthcare provider; $\mathrm{PLHIV}$, people living with $\mathrm{HIV} ; \mathrm{U}=\mathrm{U}$, undetectable equals untransmittable. 


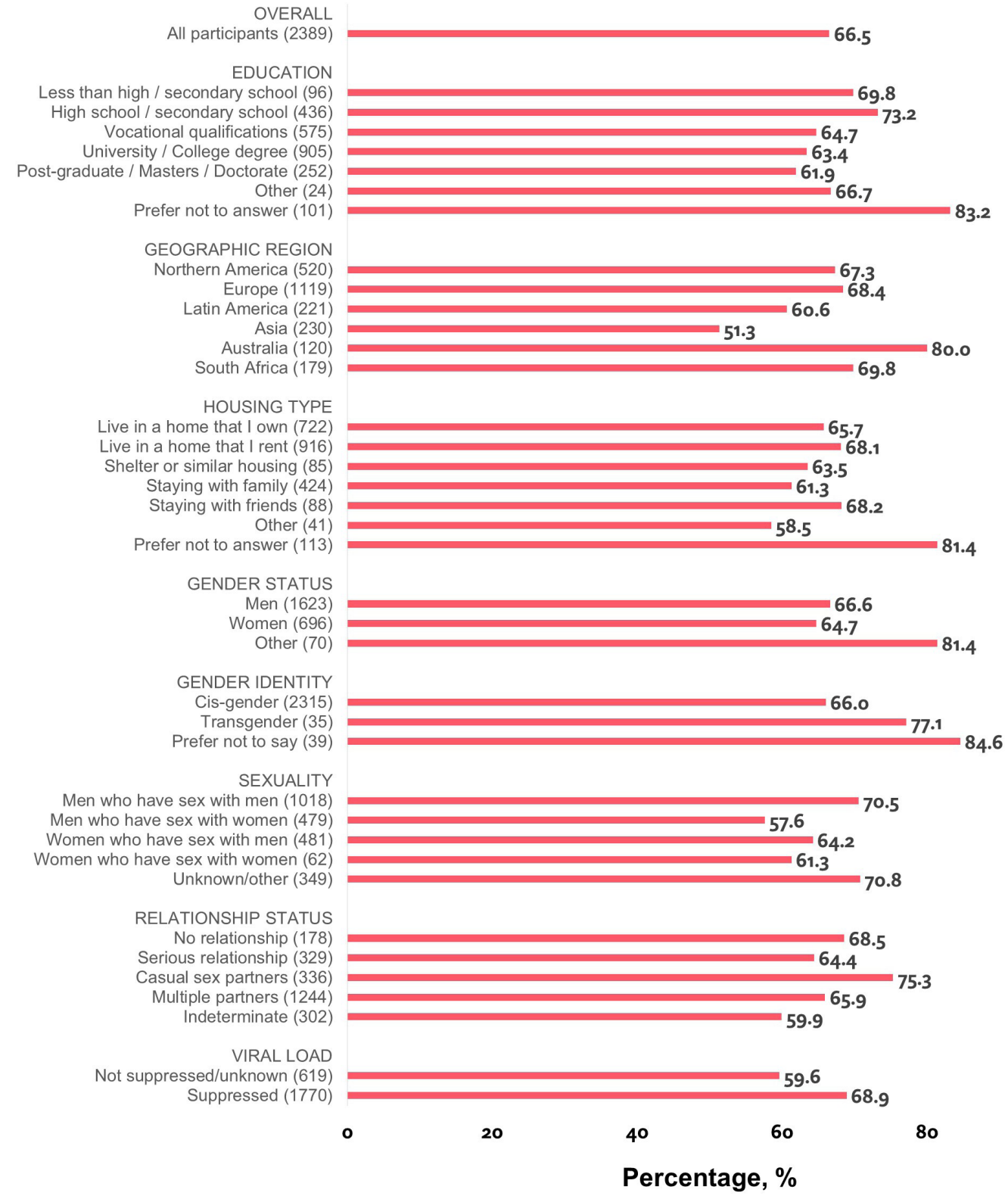

Figure 1 Percentage of people living with HIV who reported being told by their healthcare provider of 'Undetectable=Untransmittable', by selected characteristics, Positive Perspectives Study, 2019 ( $n=2389)$. Note: relationship status was assessed indirectly using a series of survey questions that asked participants with whom they had shared their HIV status. Those not in the relationship of interest selected 'not applicable'. Separate assessments were done for partner/spouse/significant other (indicated here as 'serious relationship') versus other sexual partners (indicated here as 'casual sex partners'). Those identifying they were in both relationships were classified as having multiple partners whereas those selecting 'not applicable' to both were classified as not being in any relationship. Because of skip patterns in the survey, data on relationship status could not be assessed for 302 individuals. The sum of men who have sex with men and men who have sex with women does not equal the total number of men because of individuals with missing/indeterminate information for sexual orientation; the same applies for women.

ranging from 0\% in Asia to 84.1\% (137/163) in South Africa. Other characteristics are in table 1.

\section{Percentage of PLHIV reporting past $\mathrm{U}=\mathrm{U}$ discussions with HCPs}

Overall, 66.5\% (1588/2389) reported ever discussing $U=U$ with their HCP (table 1). PLHIV who considered prevention of HIV transmission to a partner as an important treatment goal when they started ART reported significantly higher prevalence than those less concerned at ART initiation $(71.3 \%$ [836/1173] vs $61.8 \%$ [752/1216], $\mathrm{p}<0.001)$. Notably, there was no significant difference in discussing $\mathrm{U}=\mathrm{U}$ with HCPs by either year of HIV diagnosis $(p=0.886)$ or age $(p=0.119)$.
Subgroups reporting high percentages for having discussed $\mathrm{U}=\mathrm{U}$ with their HCP included people of black origins $(69.8 \%$, $192 / 275)$, those identifying as transgender (77.1\%, 27/35), with casual sex partners $(75.3 \%, 253 / 336)$, reporting viral suppression $(68.9 \%, 1219 / 1770)$ and with $\leq$ high school $(72.6 \%, 386 / 532)$ (table 1, figure 1). Prevalence by gender was 66.6\% (1081/1623) for men, $64.7 \%(450 / 696)$ for women and $81.4 \%$ (57/70) for self-reported 'Other' gender $(\mathrm{p}=0.018)$. Striking differences were observed when separating estimates for men who have sex with men $(70.5 \%, 718 / 1018)$ versus men who have sex with women $(57.6 \%, 276 / 479)(\mathrm{p}<0.001)$. Place-based differences were also noted; prevalence was highest among those whose HCP practised in a metropolitan area $(69.0 \%, 1092 / 1583)$ and whose commute 
to their HCP was $<30$ min $(71.9 \%, 625 / 869)$. Country-specific prevalence ranged from Switzerland $(87.3 \%, 48 / 55)$ to South Korea $(38.0 \%, 19 / 50)$ (online supplementary figure 1 ).

Discussions with HCPs about $\mathrm{U}=\mathrm{U}$ were correlated with other indicators of HCP engagement. Compared with those not reporting any $\mathrm{U}=\mathrm{U}$ discussions with their $\mathrm{HCP}$, those reporting a past discussion also reported significantly higher estimates for the following indicators: 'I am given enough information to be involved in making choices about my HIV treatment' $72.0 \%$ [1144/1588] vs 43.8\% [351/801]), 'My provider seeks my views about treatment before prescribing' (72.4\% [1150/1588] vs 43.7\% [350/801]), 'My provider asks me if I have any concerns about the HIV medication I am currently taking' $(74.5 \%$ [1183/1588] vs 46.6\% [373/801]), 'My provider asks me frequently about any side effects I might be experiencing with my HIV treatment' (72.8\% [1156/1588] vs 43.6\% [349/801]),
'My provider tells me about new HIV treatment options that become available' (69.3\% [1101/1588] vs 39.0\% [312/801]) and 'I feel I understand enough about my HIV treatment' $(79.0 \%$ [1254/1588] vs 56.8\% [455/801]) (all p<0.001).

\section{Differences in health outcomes with HCP versus non-HCP $\mathrm{U}=\mathrm{U}$ exposure}

Stronger associations with health outcomes were noted when PLHIV discussed $\mathrm{U}=\mathrm{U}$ with their HCP, than when exposed from non-HCP sources (tables 2 and 3). Prevalence of various health-related outcomes was as follows among the three categories of PLHIV reporting being unaware of $\mathrm{U}=\mathrm{U}$, being made aware of $U=U$ from non-HCP sources and being engaged in a past $\mathrm{U}=\mathrm{U}$ discussion with their $\mathrm{HCP}$, respectively: self-reported viral suppression (56.4\% [167/296], 76.0\% [384/505] and

Table 2 Bivariate analyses of prevalence of health outcomes by source of information about U = U, Positive Perspectives Study, 2019 ( $n=2389$ )

\begin{tabular}{|c|c|c|c|c|c|c|}
\hline Domain & Characteristic & $\begin{array}{l}\text { Overall } \\
(\mathrm{n}=2389)\end{array}$ & $\begin{array}{l}\text { Completely } \\
\text { unaware of } U=U \\
(n=296)\end{array}$ & $\begin{array}{l}\text { Aware of } \mathrm{U}=\mathrm{U} \\
\text { from non- } \\
\text { HCP sources } \\
(\mathrm{n}=505)\end{array}$ & $\begin{array}{l}\text { Informed by } \\
\text { HCP of } U=U \\
(n=1588)\end{array}$ & $P$ value \\
\hline \multirow[t]{5}{*}{ Health indicators } & Viral suppression & 74.1 & 56.4 & 76.0 & 76.8 & $<0.001$ \\
\hline & Optimal overall health & 57.6 & 44.6 & 55.0 & 60.9 & $<0.001$ \\
\hline & Optimal mental health & 57.6 & 38.2 & 53.7 & 62.5 & $<0.001$ \\
\hline & Optimal physical health & 60.1 & 45.3 & 56.2 & 64.1 & $<0.001$ \\
\hline & Optimal sexual health & 48.6 & 41.2 & 44.6 & 51.3 & 0.001 \\
\hline \multirow{7}{*}{$\begin{array}{l}\text { Attitudes and behaviours towards } \\
\text { sharing HIV status }\end{array}$} & Comfortable sharing HIV status & 29.8 & 19.6 & 26.5 & 32.7 & $<0.001$ \\
\hline & $\begin{array}{l}\text { Would be anxious or stressed if others were to } \\
\text { see their HIV medication }\end{array}$ & 45.8 & 51.0 & 50.3 & 43.3 & 0.004 \\
\hline & $\begin{array}{l}\text { Ever hid or disguised their HIV medication within } \\
\text { the past } 6 \text { months }\end{array}$ & 57.9 & 67.2 & 61.4 & 55.0 & $<0.001$ \\
\hline & $\begin{array}{l}\text { Comfortable discussing ART-related privacy } \\
\text { concerns with HCP }\end{array}$ & 52.0 & 34.8 & 45.5 & 57.2 & $<0.001$ \\
\hline & $\begin{array}{l}\text { Comfortable discussing with HCP concerns about } \\
\text { the safety of others/preventing transmission }\end{array}$ & 59.8 & 36.1 & 55.2 & 65.6 & $<0.001$ \\
\hline & Have always shared HIV status & 6.8 & 2.7 & 5.5 & 8.0 & 0.002 \\
\hline & $\begin{array}{l}\text { Missed ART dose for } 5+\text { times in past month } \\
\text { because of privacy concerns }\end{array}$ & 4.3 & 8.8 & 5.5 & 3.0 & $<0.001$ \\
\hline \multirow[t]{10}{*}{ People shared HIV status with } & $\begin{array}{l}\text { Shared HIV status with nobody besides primary } \\
\text { HIV care provider }\end{array}$ & 12.6 & 20.9 & 11.7 & 11.4 & $<0.001$ \\
\hline & 'Most of the people in my life' & 29.1 & 20.1 & 31.6 & 29.9 & 0.002 \\
\hline & Close friends & 71.1 & 56.4 & 73.9 & 72.9 & $<0.001$ \\
\hline & Parents, siblings and children & 68.1 & 60.2 & 67.4 & 69.8 & 0.006 \\
\hline & Co-workers & 33.9 & 27.5 & 39.0 & 33.4 & 0.007 \\
\hline & Wider family/circle of friends & 46.7 & 34.8 & 46.3 & 48.9 & 0.001 \\
\hline & Current family doctor not providing HIV care & 68.0 & 55.5 & 67.0 & 70.6 & $<0.001$ \\
\hline & Sexual partners & 61.1 & 50.9 & 62.0 & 62.5 & 0.005 \\
\hline & Other HCPs not providing HIV care & 60.5 & 48.4 & 62.8 & 62.0 & $<0.001$ \\
\hline & Partner/spouse/significant other & 75.8 & 69.3 & 75.8 & 77.2 & 0.025 \\
\hline \multirow[t]{10}{*}{ Reason for not sharing HIV status } & They would see or treat me different & 58.6 & 47.6 & 62.0 & 59.6 & 0.0001 \\
\hline & They might then disclose my HIV status to others & 51.8 & 48.3 & 54.7 & 51.6 & 0.210 \\
\hline & I might be excluded from activities & 38.1 & 40.2 & 39.2 & 37.4 & 0.565 \\
\hline & Being denied access to healthcare services & 18.1 & 18.2 & 24.0 & 16.2 & $<0.001$ \\
\hline & Being denied access to financial benefits & 17.7 & 20.3 & 17.8 & 17.1 & 0.427 \\
\hline & Might affect my friendships & 45.8 & 43.9 & 47.7 & 45.6 & 0.549 \\
\hline & I might lose my job & 33.9 & 34.5 & 37.6 & 32.6 & 0.115 \\
\hline & Might affect my romantic or sexual relationships & 37.3 & 27.0 & 39.2 & 38.7 & 0.001 \\
\hline & My physical safety/potential violence & 20.1 & 22.6 & 22.8 & 18.7 & 0.068 \\
\hline & Criminal prosecution & 10.1 & 10.5 & 10.5 & 9.9 & 0.899 \\
\hline
\end{tabular}

The $p$-values shown are derived from global tests (Chi-square statistics) assessing whether at least two groups are significantly different. ART, antiretroviral therapy; HCP, healthcare provider; $U=U$, undetectable equals untransmittable. 
Table 3 Binary logistic regression analyses for the relationship between awareness of $\mathrm{U}=\mathrm{U}$ and health outcomes among the entire study population, Positive Perspectives Study, 2019 ( $n=2389)$

\begin{tabular}{|c|c|c|c|c|c|}
\hline \multirow[b]{2}{*}{ Outcomes } & \multirow[b]{2}{*}{ Categories of predictor variable } & \multicolumn{2}{|l|}{ Crude analyses } & \multicolumn{2}{|l|}{ Adjusted analyses* } \\
\hline & & OR $(95 \% \mathrm{Cl})$ & $P$ value & OR $(95 \% \mathrm{Cl})$ & $P$ value \\
\hline \multirow[t]{3}{*}{ Self-reported viral suppression } & Completely unaware of $\mathrm{U}=\mathrm{U}$ & 1.00 (referent) & & 1.00 (referent) & \\
\hline & Aware from non-HCP sources & 2.45 (1.80 to 3.33$)$ & $<0.001$ & 2.10 (1.45 to 3.02$)$ & $<0.001$ \\
\hline & Informed by HCP & 2.55 (1.97 to 3.30$)$ & $<0.001$ & $2.34(1.72$ to 3.20$)$ & $<0.001$ \\
\hline \multirow[t]{3}{*}{ Suboptimal adherence } & Completely unaware of $\mathrm{U}=\mathrm{U}$ & 1.00 (referent) & & 1.00 (referent) & \\
\hline & Aware from non-HCP sources & 0.74 (0.54 to 0.99$)$ & 0.049 & 0.94 (0.68 to 1.30$)$ & 0.699 \\
\hline & Informed by HCP & $0.46(0.35$ to 0.60$)$ & $<0.001$ & $0.59(0.44$ to 0.78$)$ & $<0.001$ \\
\hline \multirow[t]{3}{*}{ Optimal overall health } & Completely unaware of $\mathrm{U}=\mathrm{U}$ & 1.00 (referent) & & 1.00 (referent) & \\
\hline & Aware from non-HCP sources & 1.52 (1.14 to 2.03$)$ & 0.004 & 1.51 (1.12 to 2.03$)$ & 0.007 \\
\hline & Informed by HCP & 1.93 (1.51 to 2.49$)$ & $<0.001$ & 1.94 (1.49 to 2.51$)$ & $<0.001$ \\
\hline \multirow[t]{3}{*}{ Optimal mental health } & Completely unaware of $\mathrm{U}=\mathrm{U}$ & 1.00 (referent) & & 1.00 (referent) & \\
\hline & Aware from non-HCP sources & 1.88 (1.40 to 2.51$)$ & $<0.001$ & 1.79 (1.32 to 2.43$)$ & $<0.001$ \\
\hline & Informed by HCP & 2.70 (2.09 to 3.48$)$ & $<0.001$ & 2.50 (1.91 to 3.26$)$ & $<0.001$ \\
\hline \multirow[t]{3}{*}{ Optimal physical health } & Completely unaware of $\mathrm{U}=\mathrm{U}$ & 1.00 (referent) & & 1.00 (referent) & \\
\hline & Aware from non-HCP sources & 1.55 (1.16 to 2.07$)$ & 0.003 & 1.44 (1.07 to 1.94$)$ & 0.018 \\
\hline & Informed by HCP & 2.16 (1.68 to 2.77$)$ & $<0.001$ & 1.95 (1.50 to 2.54$)$ & $<0.001$ \\
\hline \multirow[t]{3}{*}{ Optimal sexual health } & Completely unaware of $\mathrm{U}=\mathrm{U}$ & 1.00 (referent) & & 1.00 (referent) & \\
\hline & Aware from non-HCP sources & $1.15(0.86$ to 1.53$)$ & 0.358 & $1.13(0.83$ to 1.53$)$ & 0.441 \\
\hline & Informed by HCP & 1.50 (1.17 to 1.93$)$ & 0.001 & 1.48 (1.14 to 1.92$)$ & 0.004 \\
\hline \multirow{3}{*}{$\begin{array}{l}\text { Comfortable sharing } \\
\text { HIV status }\end{array}$} & Completely unaware of $\mathrm{U}=\mathrm{U}$ & 1.00 (referent) & & 1.00 (referent) & \\
\hline & Aware from non-HCP sources & 1.48 (1.05 to 2.10$)$ & 0.027 & 1.58 (1.10 to 2.26$)$ & 0.013 \\
\hline & Informed by HCP & 1.99 (1.47 to 2.70$)$ & $<0.001$ & 2.15 (1.57 to 2.95$)$ & $<0.001$ \\
\hline \multirow[t]{3}{*}{ Always share HIV status } & Completely unaware of $\mathrm{U}=\mathrm{U}$ & 1.00 (referent) & & 1.00 (referent) & \\
\hline & Aware from non-HCP sources & $2.11(0.95$ to 4.70$)$ & 0.067 & $2.10(0.93$ to 4.77$)$ & 0.075 \\
\hline & Informed by HCP & $3.13(1.51$ to 6.46$)$ & 0.002 & 2.99 (1.42 to 6.28$)$ & 0.004 \\
\hline \multirow{3}{*}{$\begin{array}{l}\text { Shared HIV status with others besides HIV care } \\
\text { provider }\end{array}$} & Completely unaware of $\mathrm{U}=\mathrm{U}$ & 1.00 (referent) & & 1.00 (referent) & \\
\hline & Aware from non-HCP sources & 2.00 (1.36 to 2.96$)$ & $<0.001$ & 1.67 (1.11 to 2.52$)$ & 0.014 \\
\hline & Informed by HCP & 2.06 (1.50 to 2.84$)$ & $<0.001$ & 1.74 (1.24 to 2.45$)$ & 0.001 \\
\hline \multirow[t]{3}{*}{ Ever disguised/hid HIV medicines in past 6 months } & Completely unaware of $\mathrm{U}=\mathrm{U}$ & 1.00 (referent) & & 1.00 (referent) & \\
\hline & Aware from non-HCP sources & $0.77(0.57$ to 1.05$)$ & 0.098 & $0.87(0.63$ to 1.21$)$ & 0.417 \\
\hline & Informed by HCP & $0.60(0.46$ to 0.78$)$ & $<0.001$ & $0.65(0.49$ to 0.86$)$ & 0.003 \\
\hline \multirow[t]{3}{*}{ Comfortable discussing privacy issues with HCP } & Completely unaware of $\mathrm{U}=\mathrm{U}$ & 1.00 (referent) & & 1.00 (referent) & \\
\hline & Aware from non-HCP sources & 1.57 (1.17 to 2.11$)$ & 0.003 & 1.41 (1.04 to 1.91$)$ & 0.029 \\
\hline & Informed by HCP & 2.51 (1.94 to 3.25$)$ & $<0.001$ & 2.50 (1.91 to 3.28$)$ & $<0.001$ \\
\hline \multirow{3}{*}{$\begin{array}{l}\text { Comfortable discussing concerns about } \\
\text { transmission with HCP }\end{array}$} & Completely unaware of $\mathrm{U}=\mathrm{U}$ & 1.00 (referent) & & 1.00 (referent) & \\
\hline & Aware from non-HCP sources & 2.18 (1.62 to 2.93$)$ & $<0.001$ & $1.93(1.42$ to 2.62$)$ & $<0.001$ \\
\hline & Informed by HCP & $3.37(2.60$ to 4.37$)$ & $<0.001$ & $3.27(2.49$ to 4.29$)$ & $<0.001$ \\
\hline \multirow{3}{*}{$\begin{array}{l}\text { Missed ART for } \geq 5 \text { times in past month because of } \\
\text { privacy concerns }\end{array}$} & Completely unaware of $\mathrm{U}=\mathrm{U}$ & 1.00 (referent) & & 1.00 (referent) & \\
\hline & Aware from non-HCP sources & $0.61(0.35$ to 1.06$)$ & 0.080 & $0.83(0.47$ to 1.48$)$ & 0.533 \\
\hline & Informed by HCP & $0.32(0.20$ to 0.53$)$ & $<0.001$ & $0.41(0.25$ to 0.69$)$ & 0.001 \\
\hline
\end{tabular}

${ }^{*}$ Adjusted for ethnicity, region, age, sexual orientation, diagnosis year, domicile and education.

$A R T$, antiretroviral therapy; HCP, healthcare provider; $\mathrm{U}=\mathrm{U}$, undetectable equals untransmittable.

$76.8 \%$ [1219/1588]), optimal overall health (44.6\% [132/296], $55.0 \%[278 / 505]$ and $60.9 \%[967 / 1588])$, optimal sexual health (41.2\% [122/296], 44.6\% [225/505] and 51.3\% [815/1588]), never shared their HIV status with anyone besides their primary HIV care providers $(20.9 \%$ [62/296], 11.7\% [59/505] and $11.4 \%$ [181/1588]), perceived comfort discussing concerns regarding HIV transmission with HCP (36.1\% [107/296], 55.2\% [279/505] and 65.6\% [1042/1588]) and suboptimal adherence (35.8\% [106/296], 29.1\% [147/505] and 20.3\% [322/1588]) (all $\mathrm{p}<0.05$, table 2 ).

Results remained consistent within multivariable analyses (table 3). Those who reported discussing $\mathrm{U}=\mathrm{U}$ with their HCP had more favourable outcomes compared with those unaware of $\mathrm{U}=\mathrm{U}$, for: suboptimal adherence (adjusted $\mathrm{OR}(\mathrm{AOR})=0.59$, $95 \%$ CI 0.44 to 0.78 ); optimal sexual health (AOR $=1.48,95 \%$ CI 1.14 to 1.92 ) and reporting they 'always shared' their HIV status (AOR $=2.99,95 \%$ CI 1.42 to 6.28 ). For several positive outcomes, when compared with those unaware of $\mathrm{U}=\mathrm{U}$, significantly higher odds were seen among both those made aware of $\mathrm{U}=\mathrm{U}$ from non-HCP sources, as well as those reporting a past $\mathrm{U}=\mathrm{U}$ discussion with their HCP although stronger associations were noted for the latter. These outcomes included: viral suppression $(\mathrm{AOR}=2.10$ and 2.34, non-HCP and HCP, respectively); optimal overall health $(\mathrm{AOR}=1.51$ and 1.94 , respectively); optimal mental health $(\mathrm{AOR}=1.79$ and 2.50 , respectively); comfortable sharing HIV status with others $(\mathrm{AOR}=1.58$ and 
2.15, respectively); ever shared HIV status with people other than their primary HIV HCPs $(\mathrm{AOR}=1.67$ and 1.74 , respectively); comfortable discussing ART-related privacy concerns with $\mathrm{HCP}(\mathrm{AOR}=1.41$ and 2.50 , respectively) and comfortable discussing with HCPs their concerns regarding HIV transmission $(\mathrm{AOR}=1.93$ and 3.27, respectively) (all $\mathrm{p}<0.05)$.

\section{Differences in health outcomes by subgroups exposed to the $\mathrm{U}=\mathrm{U}$ message}

Within four mutually exclusive strata of age and gender, $\geq 50$-year-old men reported the highest prevalence of sharing their HIV status with family, friends or co-workers; conversely, $<50$-year-old women were least likely to share their HIV status among their social contacts (online supplementary figure 2).

The observed strength of association between exposure to the $\mathrm{U}=\mathrm{U}$ message and health outcomes varied by age and gender. For example, compared with those unaware of $\mathrm{U}=\mathrm{U}$, discussing $\mathrm{U}=\mathrm{U}$ with an $\mathrm{HCP}$ was significantly associated with higher odds of optimal sexual health among $<50$-year-olds $(\mathrm{AOR}=1.79$; $95 \%$ CI 1.33 to 2.42 ), but non-significant among $\geq 50$-year-olds within adjusted analyses. Conversely, discussing $\mathrm{U}=\mathrm{U}$ with an HCP was associated with over five times higher odds of sharing HIV status with other people among $\geq 50$-year-olds $(\mathrm{AOR}=5.58$; $95 \%$ CI 2.59 to 12.03 ) whereas a more attenuated association was observed among $<50$-year-olds $(\mathrm{AOR}=1.60,95 \% \mathrm{CI} 1.10$ to 2.33) (online supplementary table 3 ).

\section{DISCUSSION}

Overall, $66.5 \%$ of PLHIV in our study had discussed $\mathrm{U}=\mathrm{U}$ with their HCP. We saw no significant difference between PLHIV diagnosed prior to 2017 vs 2017 or later in being told of $\mathrm{U}=\mathrm{U}$ by their $\mathrm{HCP}^{24}$. Indeed, almost a decade before the launch of the $\mathrm{U}=\mathrm{U}$ campaign, ${ }^{24}$ the Swiss Federal Commission for HIV/AIDS first endorsed a similar message of undetectable equals uninfectious in $2008 .^{25}{ }^{26}$ The global $\mathrm{U}=\mathrm{U}$ campaign has however allowed for universal endorsement of this message. A possible explanation for the non-significant finding by time of diagnosis is that patient education about $\mathrm{U}=\mathrm{U}$ may be occurring at multiple periods throughout the course of treatment other than just at time of diagnosis, thus explaining the similar rates of ever receiving $\mathrm{U}=\mathrm{U}$ information from an $\mathrm{HCP}$ regardless of when diagnosed. We also observed that people of lower educational status were more likely to report receiving information on $\mathrm{U}=\mathrm{U}$ from their HCP, possibly reflecting lack of other sources. Proactively discussing $\mathrm{U}=\mathrm{U}$ at different points, including at the time of diagnosis, initiating ART and during routine follow-up consultations can empower PLHIV. ${ }^{27}$ Even brief counselling of $2-3 \mathrm{~min}$ has been shown to be effective with other behavioural interventions such as smoking cessation counselling. ${ }^{28}$

Information provided by HCPs may be deemed more credible than other media which may explain our observation of stronger associations with positive health outcomes among those who had the opportunity to discuss $\mathrm{U}=\mathrm{U}$ with an $\mathrm{HCP}$ versus those who accessed similar information from non-HCP sources. PLHIV who were concerned about transmitting HIV reported significantly higher prevalence of receiving information on $\mathrm{U}=\mathrm{U}$ from their HCP, suggesting patient-initiated discussions. At the same time, however, PLHIV who reported having $\mathrm{U}=\mathrm{U}$ discussion with their HCPs also were more likely to be engaged by their HCPs on other fronts, including discussing new treatment options as well as their management plan before prescribing treatment, suggesting a stronger patient/doctor relationship. Patients that are actively engaged with their HCP may proactively invite these topics of discussion compared with patients less engaged; however, the onus of these discussions should not be placed on the patient. HCP initiation of these sensitive topics in patients that have difficulty engaging may also help empower patients. Our findings indicate that directly addressing sexual health and $\mathrm{U}=\mathrm{U}$ is associated with significantly better health outcomes.

PLHIV face many emotional/psychological challenges, including self-stigma, which may impact their ability, desire or comfort level with sharing their emotional concerns with HCPs. Creating an enabling, non-judgmental and stigma-free environment where PLHIV feel encouraged to discuss personal challenges is critical. Independent of duration of diagnosis, exposure to $\mathrm{U}=\mathrm{U}$ message was associated with higher odds of better social interactions among older adults but higher odds of optimal sexual health among younger ones, suggesting differences in perceived priorities and life experiences. Tailored communication regarding the benefits of $\mathrm{U}=\mathrm{U}$ among PLHIV could improve acceptability of the $\mathrm{U}=\mathrm{U}$ message.

Previous research has identified some degree of misunderstanding, confusion and even suspicion among those informed of ' $U=U$ '; inconsistent HCP practices in relation to $U=U$ have also been reported previously. ${ }^{2930}$ A survey of 270 members of the British HIV Association found variations in timing of delivery and the substance of the message itself. For example, varying terminologies were reported to describe the risk of HIV transmission such as 'zero risk/no risk', 'next to zero', 'negligible' or 'extremely low'. This can be confusing as 'negligible' is often interpreted as still a non-zero risk. ${ }^{27}{ }^{29}$ HCPs should use clear, unambiguous language to convey $\mathrm{U}=\mathrm{U}$, while reinforcing to patients that the $\mathrm{U}=\mathrm{U}$ message is not about discouraging condom use or responsible sexual behaviour; rather, it is about providing assurance about their sometimes unexpressed fear of infecting others-a key cause of stigma, shame and poor psychological well-being. ${ }^{923}$ Recommending $\mathrm{U}=\mathrm{U}$ discussions as a standard in clinical guidelines can help the uniformity of information dissemination, endorse the $\mathrm{U}=\mathrm{U}$ message internationally, and help improve HIV care beyond viral load end points.

Opportunities exist to enhance dissemination of this key information considering that a third of PLHIV were not informed by their provider. Implementation research and training is needed to better understand how to optimise delivery of the $\mathrm{U}=\mathrm{U}$ message in clinical settings. Research is also needed on the effectiveness of $\mathrm{U}=\mathrm{U}$ counselling when delivered by a variety of healthcare professionals. Understanding the unique challenges to delivering (among $\mathrm{HCPs}$ ) or believing (among PLHIV) the $\mathrm{U}=\mathrm{U}$ message can inform public health practice, programmes and policies.

\section{Strengths and limitations}

The strengths of this study include the use of a standardised protocol in 25 countries and a large sample size which increases precision of estimates. Nonetheless, limitations exist. The non-probabilistic sampling and web-based survey administration may limit generalisability while the self-reported measures may be subject to misreporting and other measurement errors. For example, there could be underestimation of the percentage informed about $\mathrm{U}=\mathrm{U}$ in clinical settings because of poor recall, or if PLHIV were informed by someone other than their primary HIV care provider. We could not explore non-HCP sources of $\mathrm{U}=\mathrm{U}$ information in great detail because of limited information. Misclassification of $\mathrm{U}=\mathrm{U}$ exposure status could also arise from dichotomisation of the Likert-type scale, but the effect of this misclassification is likely to be conservatively towards the null (ie, individuals with some exposure classified as being non-exposed). Finally, only associations can be drawn because of the cross-sectional design. 
Key messages

- A third of people living with HIV (PLHIV) in this international study reported not having had discussions with their healthcare provider (HCP) about 'Undetectable equals Untransmittable' ('U=U').

- PLHIV who reported discussing with their HCP about $U=U$ had more favourable health outcomes than those not informed of $\mathrm{U}=\mathrm{U}$; for example, they had $41 \%$ lower odds of reporting suboptimal adherence and $48 \%$ higher odds of optimal sexual health.

- While U=U-related information accessed from non-HCP sources was beneficial as well, the observed associations were attenuated relative to those seen with actually discussing $\mathrm{U}=\mathrm{U}$ with HCPs.

- $\mathrm{U}=\mathrm{U}$ discussions between HCPs and patients should be considered in standard of care guidelines to help improve health-related outcomes of PLHIV and benefit public health.

\section{CONCLUSIONS}

Overall, only $66.5 \%$ of all PLHIV reported having discussed $\mathrm{U}=\mathrm{U}$ with their HCP and disparities by region and other characteristics were observed. Discussing $\mathrm{U}=\mathrm{U}$ with an HCP was significantly associated with favourable health outcomes, including greater adherence, better viral control, optimal health (including sexual health), greater willingness to share HIV status with others, and increased comfort discussing with HCP concerns about HIV transmission and privacy. Increased dissemination of the $\mathrm{U}=\mathrm{U}$ message in clinical settings can benefit public health.

\author{
Author affiliations \\ ${ }^{1}$ ViiV Healthcare, Brentford, UK \\ ${ }^{2}$ Prevention Access Campaign, New York City, New York, USA \\ ${ }^{3}$ International Council of AIDS Service Organizations (ICASO), Toronto, Ontario, \\ Canada \\ ${ }^{4}$ Global Action for Trans* Equality (GATE), Belize City, Belize \\ ${ }^{5}$ ViiV Healthcare, Research Triangle Park, North Carolina, USA \\ ${ }^{6}$ Positively UK, London, UK \\ ${ }^{7}$ AIDS Center Foundation, Moscow, Russia, Moscow, Russian Federation \\ ${ }^{8}$ European AIDS Treatment Group, Rome, Lazio, Italy, Rome, Italy \\ ${ }^{9}$ Instituto Cultural Barong, São Paulo, Brazil, São Paulo, Brazil \\ ${ }^{10}$ Johns Hopkins School of Medicine, Baltimore, Maryland, USA
}

\section{Handling editor Anna Maria Geretti}

Twitter Anton Eremin @DrAntonEremin and Giulio Maria Corbelli @giuliomcorbelli

Special Acknowledgement Data analyses and medical writing services were provided by Zatum LLC.

Contributors CO, PdIR, BY and NVdV conceptualised the study. All authors contributed to study design, analyses, drafting of the manuscript and substantial revisions. All authors gave final approval for the manuscript to be submitted.

Funding This work was supported by ViiV Healthcare.

Competing interests The following authors are employees of ViiV Healthcare: $\mathrm{CO}$, PdIR, BY and NVdV.

Patient consent for publication Not required

Ethics approval Ethical review was provided by the Pearl Institutional Review Board (no. 18-0 80 622). In addition, specific approval for South Africa was obtained from the Sefako Makgatho Research Ethics Committee (no. SMUREC/M/223/2019).

Provenance and peer review Not commissioned; externally peer reviewed.

Data availability statement Requests to access the primary data analysed should be directed to the corresponding author.

Open access This is an open access article distributed in accordance with the Creative Commons Attribution Non Commercial (CC BY-NC 4.0) license, which permits others to distribute, remix, adapt, build upon this work non-commercially, and license their derivative works on different terms, provided the original work is properly cited, appropriate credit is given, any changes made indicated, and the use is non-commercial. See: http://creativecommons.org/licenses/by-nc/4.0/.

\section{ORCID iD}

Anton Eremin http://orcid.org/0000-0001-6232-1764

\section{REFERENCES}

1 The Joint United Nations Programme on HIV/AIDS (UNAIDS). Global HIV \& AIDS statistics - 2019 fact sheet, 2019. Available: https://www.unaids.org/en/resources/ fact-sheet [Accessed 10 Feb 2020].

2 AVERT. Global HIV and AIDS statistics. Available: https://www.avert.org/global-hivand-aids-statistics

3 Prevention Access Campaign. About, 2017. Available: https://www.preventionaccess. org/about [Accessed 6 Jan 2020].

4 Prevention Access Campaign. Consensus statement, 2019. Available: https://www. preventionaccess.org/consensus [Accessed 6 Jan 2020].

5 Centers for Disease Control and Prevention. Effectiveness of prevention strategies to reduce the risk of acquiring or transmitting HIV, 2019. Available: https://www.cdc.gov/ hiv/risk/estimates/preventionstrategies.html [Accessed 6 Jan 2020].

6 Cohen MS, Chen YQ, McCauley M, et al. Antiretroviral therapy for the prevention of HIV-1 transmission. N Engl J Med 2016;375:830-9.

7 Rodger AJ, Cambiano V, Bruun T, et al. Sexual activity without condoms and risk of HIV transmission in serodifferent couples when the HIV-positive partner is using suppressive antiretroviral therapy. JAMA 2016;316:171-81.

8 Bavinton BR, Pinto AN, Phanuphak N, et al. Viral suppression and HIV transmission in serodiscordant male couples: an international, prospective, observational, cohort study. Lancet HIV 2018;5:e438-47.

9 Earnshaw VA, Chaudoir SR. From conceptualizing to measuring HIV stigma: a review of HIV stigma mechanism measures. AIDS Behav 2009;13:1160.

10 Fay H, Baral SD, Trapence G, et al. Stigma, health care access, and HIV knowledge among men who have sex with men in Malawi, Namibia, and Botswana. AIDS Behav 2011;15:1088-97.

11 Rueda S, Mitra S, Chen S, et al. Examining the associations between HIV-related stigma and health outcomes in people living with HIVIAIDS: a series of meta-analyses. BMJ Open 2016;6:e011453.

12 Philbin MM, Hirsch JS, Wilson PA, et al. Structural barriers to HIV prevention among men who have sex with men (MSM) in Vietnam: diversity, stigma, and healthcare access. PLoS One 2018;13:e0195000

13 Australasian Society for HIV, Sexual Health and Viral Hepatitis Medicine (ASHM). Guidelines for health care providers for providing advice regarding $\mathrm{U}=\mathrm{U}$ (undetectable = untransmittible). Available: https://www.ashm.org.au/HIV/UequalsU.pdf [Accessed 04 Apr 2020].

14 Hesse BW, Nelson DE, Kreps GL, et al. Trust and sources of health information: the impact of the Internet and its implications for health care providers: findings from the first health information national trends survey. Arch Intern Med 2005;165:2618-24.

15 U.S. Preventive Services Task Force. Published recommendations. Available: https:// www.uspreventiveservicestaskforce.org/BrowseRec/Index

16 World Health Organization Regional Office for Europe. HIV/AIDS surveillance in Europe 2018, 2017. Available: https://www.ecdc.europa.eu/sites/default/files/ documents/hiv-aids-surveillance-europe-2018.pdf [Accessed 02 Nov 2020].

17 Centers for Disease Control and Prevention. Hiv surveillance report, 2018 (preliminary), 2019. Available: http://www.cdc.gov/hiv/library/reports/hiv-surveillance. html [Accessed 02 Nov 2020].

18 U.S Department of Health and Human Services. U.S. statistics.. Available: https://www. hiv.gov/hiv-basics/overview/data-and-trends/statistics [Accessed 2/12/2020].

19 Kirby Institute. Hiv in Australia: annual surveillance short report 2018. Sydney: Kirby Institute, UNSW Sydney, 2018. https://kirby.unsw.edu.au/sites/default/files/kirby/ report/supplHIV2018_content_20180920r.pdf

20 Camacho-Gonzalez AF, Wallins A, Toledo L, et al. Risk factors for HIV transmission and barriers to HIV disclosure: metropolitan Atlanta youth perspectives. AIDS Patient Care STDS 2016;30:18-24.

21 Marsh K, Eaton JW, Mahy M, et al. Global, regional and country-level 90-9090 estimates for 2018: assessing progress towards the 2020 target. AIDS 2019;33:\$213-26.

22 Stutterheim SE, Pryor JB, Bos AER, et al. Hiv-Related stigma and psychological distress: the harmful effects of specific stigma manifestations in various social settings. AIDS 2009:23:2353-7.

23 NAM aidsmap. NAM endorses undetectable equals Untransmittable $(U=U)$ consensus statement, 2017. Available: http://www.aidsmap.com/news/feb-2017/nam-endorsesundetectable-equals-untransmittable-uu-consensus-statement\#_ednref1 [Accessed 6 Jan 2020].

24 The Lancet HIV. U=U taking off in 2017. Lancet HIV 2017;4:e475.

25 Vernazza P, Hirschel B, Bernasconi E, et al. Les personnes séropositives ne souffrant d'aucune autre MST et suivant un traitment antirétroviral efficace ne transmettent pas le VIH par voie sexuelle. Bulletin des médecins suisses 2008;89.

26 AIDS Map. Swiss experts say individuals with undetectable viral load and no STI cannot transmit HIV during sex. Available: http://www.aidsmap.com/news/jan-2008/ 
swiss-experts-say-individuals-undetectable-viral-load-and-no-sti-cannot-transmit-hiv [Accessed 6 Apr 2020].

27 British HIV Association. BHIVA encourages universal promotion of Undetectable=Unstransmittable $(U=U)$, 2018. Available: https://www.bhiva.org/ BHIVA-encourages-universal-promotion-of-U-U [Accessed 6 Jan 2020].

28 Fiore MC. US public health service clinical practice guideline: treating tobacco use and dependence. Respir Care 2000;45:1200-62.
29 Gupta N, Gilleece Y, Orkin C. Implementing $U=U$ in clinical practice: results of a British HIV association members survey [published online ahead of print, 2020 Mar 5]. Sex Transm Infect 2020:sextrans-2020-054462.

30 Rendina HJ, Cienfuegos-Szalay J, Talan A, et al. Growing acceptability of Undetectable= Untransmittable but widespread misunderstanding of transmission risk: findings from a very large sample of sexual minority men in the United States. $J$ Acquir Immune Defic Syndr 2019;83:215-22. 